\title{
La longue marche des crêtes neurales
}

\section{Jean-Loup Duband}

J.L. Duband : Laboratoire de biologie moléculaire et cellulaire du développement. UMR 7622, Cnrs et Université Pierre-etMarie-Curie, 9, quai Saint-Bernard, 75252 Paris Cedex 05, France.

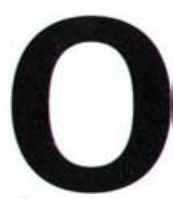

n a longtemps sous-estimé l'importance de la migration dans l'élaboration du plan d'organisation de I'animal au cours du développement embryonnaire. Elle était souvent confinée au rôle marginal de finition, ou bien on considérait qu'elle ne concernait qu'un nombre restreint de cellules. Ce n'est que relativement récemment $\mathrm{qu}^{\prime}$ on a $\mathrm{pu}$ apprécier son importance dès les étapes les plus précoces du développement embryonnaire. Ainsi, chez tous les vertébrés et nombre d'invertébrés, la construction du feuillet mésodermique à l'origine de tout le squelette, des muscles et des organes internes n'a lieu que grâce à un phénomène de migration de grande ampleur à partir du feuillet supérieur de l'embryon: c'est la gastrulation. Le passage évolutif dans le règne animal d'espèces primitives à deux feuillets embryonnaires simples vers des espèces beaucoup plus élaborées à trois feuillets a donc nécessité la mise en place, et son contrôle spatio-temporel, de la migration de populations entières de cellules et non plus de quelques cellules isolées. Après la gastrulation, d'autres migrations contribuent à l'organogenèse: par exemple, lors de la formation du système nerveux périphérique, durant l'établissement de la musculature des membres et des viscères, ou bien encore pendant la formation du rein. Enfin, des populations cellulaires (celIules germinales et cellules lymphoïdes) vont entreprendre des migrations pour aller coloniser des organes dans lesquels elles vont terminer leur différenciation. Entre autres intérêts, ces événements migratoires vont permettre à des populations cellulaires issues d'une région donnée de l'embryon de traverser des environnements variés et, par là, de recevoir des signaux qui vont orienter leur différenciation et être responsables de la ségrégation de lignages cellulaires différents. Inversement, ils permettent aussi à certaines cellules issues de régions différentes de l'embryon de se loger dans un territoire cible précis où elles vont interagir localement et échanger des informations avant de s'engager dans des programmes de différenciation cohérents et concertés.

Au cours du développement embryonnaire, tous les modes de migration sont observés: cellules isolées, en amas ou en nappe; migration divergente vers des tissus variés ou convergente vers un site unique; transport passif par les fluides ou migration active à travers une matrice extracellulaire ou en contact avec d'autres cellules; migration invasive avec traversée de parois vasculaires; migration orientée par chimiotactisme ou «au hasard». Tous ces évé- 
nements migratoires ont fait l'objet d'études plus ou moins poussées, chacun présentant des spécificités intéressantes dans la chronologie des événements cellulaires; mais il est un système cellulaire qui a de tout temps eu les faveurs des chercheurs: les crêtes neurales. A cela, plusieurs raisons: tout d'abord, c'est un phénomène massif qui concerne tout l'embryon, tant au niveau crânien que troncal. II s'étend sur une durée d'un à deux jours, voire plus pour certaines régions. Il est à l'origine de la formation $d^{\prime}$ un très grand nombre de types cellulaires, d'un intérêt primordial notamment pour le développement cranio-facial. II résume à lui seul presque l'intégralité du registre des événements cellulaires qui ponctuent la migration. Sur le plan expérimental, il est possible chez les espèces ovipares (oiseaux, amphibiens, poissons) d'accéder directement dans l'embryon à la population des cellules des crêtes neurales au début de la migration, ce qui est pratique pour des manipulations in situ. Enfin, ce système peut être transposé dans des conditions de culture in vitro qui reproduisent assez fidèlement la dynamique du développement in vivo. L'histoire naturelle des crêtes neurales est résumée sur la figure 1. Elle commence entre le premier et le deuxième jour de développement chez le poulet, le huitième et le neuvième chez la souris et au cours de la troisième semaine de gestation chez I'homme. Les cellules qui les composent sont issues de la région de l'embryon à la frontière entre le futur épiderme (I'ectoderme) et le futur système nerveux central (le tube neural), au moment où ceux-ci se séparent I'un de I'autre. Elles s'appellent crêtes neurales parce que, chez certains amphibiens chez lesquels elles ont été décrites pour la première fois, elles forment des protubérances transitoires caractéristiques sur le sommet du tube neural, peu après leur constitution. Après une phase de spécification pendant laquelle elles reçoivent les signaux de l'environnement qui vont dicter leur devenir, les cellules des crêtes neurales se dissocient du tube neural par un mécanisme faisant intervenir une transition épithélio-mésenchymateuse : c'est la phase de délamination. Chez la plupart des espèces, cette phase est aussitôt suivie par la migration proprement dite qui, une fois terminée, est prolongée par un regroupement des cellules, prélude à leur différenciation terminale. La liste des dérivés cellulaires issus des crêtes neurales est impressionnante et, en cela, révélatrice du potentiel que recèle la migration pour créer une grande diversité cellulaire à partir d'une population unique: neurones, cellules gliales et cellules de Schwann du système nerveux périphérique, cellules pigmentaires, cellules endocrines, ou encore tissus conjonctifs, cartilagineux, osseux et musculaires dans la région crânienne qui englobe la face, le crâne et le cou [1]. La somme des données accumulées au cours des deux dernières décennies permet maintenant une meilleure compréhension des mécanismes moléculaires qui régissent le développement des crêtes neurales, et laisse entrevoir des perspectives plus générales sur l'étude des processus fondamentaux de la migration cellulaire, tant dans les cas physiologiques normaux que pathologiques.

\section{Comment les suivre? À la recherche des marqueurs des crêtes neurales ou la quête du Graal}

A l'exception de l'embryon de poisson-zèbre, qui offre des propriétés de transparence exceptionnelles, et de l'amphibien axolotl, chez lequel les

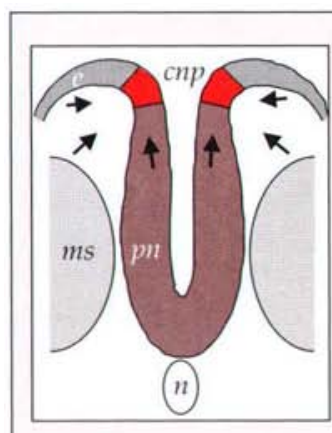

(1) Spécification

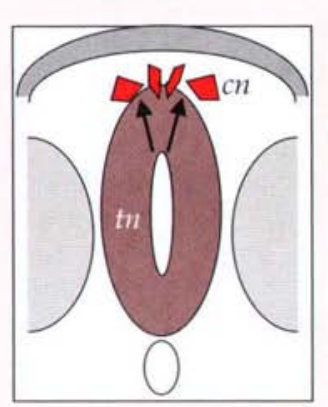

(2) Délamination

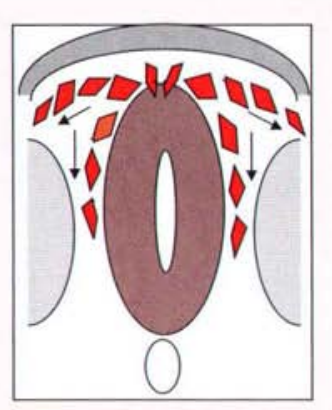

(3) Migration

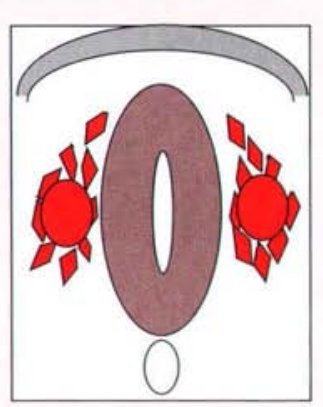

(4) Agrégation

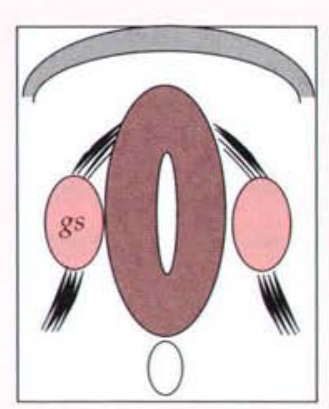

(5) Différenciation

Figure 1. Les principales étapes du développement des cellules des crêtes neurales. Les cellules du primordium des crêtes neurales (cnp, en rouge) apparaissent à la frontière entre l'ectoderme superficiel (e, en gris) et la plaque neurale (pn, en bistre) en fin de neurulation, suite à un phénomène complexe de spécification impliquant des inductions et des informations de position (grandes flèches) provenant de l'ectoderme, de la plaque neurale et du mésoderme somitique (ms) voisins. La spécification est suivie de la délamination des cellules des crêtes neurales (cn) du tube neural (tn) et de leur migration le long de voies précises (flèches fines). Une fois rejoints les territoires cibles, les cellules cessent leur migration et se regroupent en amas, préludes à la différenciation en ganglions du système nerveux périphérique. À quelques petites nuances près (notamment le stade de la fermeture de la plaque neurale en tube neural), cette séquence d'événements est la même quel que soit le niveau de l'axe antéro-postérieur considéré pour toutes les espèces de vertébrés supérieurs, des poissons aux mammifères. gs: ganglion sensoriel; $n$ : notochorde. 
précurseurs mélanocytaires sont précocément pigmentés, il n'est quasiment pas possible de visualiser les crêtes neurales en migration dans l'embryon. D'où le développement: de techniques permettant de pister les cellules. La mise au point et l'application des chimères caille-poule développées par Nicole Le Douarin il y a trente ans ont représenté une percée technologique décisive, car elle a permis d'établir avec précision la liste et la cartographie des dérivés des crêtes neurales et de préciser les parcours de migration [2]. Par la suite, I'utilisation de traceurs fluorescents tels que le Dil ou du dextran couplé à la rhodamine, injectés dans l'embryon, a permis d'affiner les connaissances dans des espèces dans lesquelles l'approche par les chimères n'était pas possible, et a aussi fourni des informations sur la dynamique des événements à l'échelon d'une population cellulaire restreinte $[3,4]$. En marge de ces approches expérimentales qui ont l'inconvénient d'être délicates, la recherche de marqueurs moléculaires des crêtes neurales a été et continue d'être un enjeu majeur de ces dernières années. En effet, on ne dispose pas encore du marqueur parfait (si tant est qu'un tel marqueur existe!) qui permette de distinguer chez différentes espèces animales l'intégralité de la population des crêtes neurales à tous les niveaux, depuis leur spécification jusqu'à leur différenciation. C'est chez le poulet ou la caille que I'on possède les meilleurs outils: I'anticorps HNK-1, notamment, est couramment employé pour suivre les crêtes neurales en migration à tous les niveaux axiaux ainsi que leurs principaux dérivés nerveux. Cet anticorps reconnaît un groupement glycosidique porté par des lipides ou des glycoprotéines de surface portant des domaines immunoglobulines. Cependant, c'est un marqueur médiocre des mélanoblastes et des dérivés non-nerveux des crêtes neurales crâniennes et, autres inconvénients, il peut être exprimé par des cellules ne dérivant pas des crêtes neurales et il n'est utilisable ni chez l'amphibien xénope, ni chez la souris [5]. Chez cette dernière, d'ailleurs, on est loin de posséder un marqueur aussi "universel» qu'HNK-1 et on doit se contenter de plusieurs marqueurs souvent peu spé- cifiques qui ne permettent de suivre que certains lignages issus des crêtes neurales à des niveaux axiaux et à des stades de la migration donnés [6]. On citera pêle-mêle: AP2, qui permet de repérer les crêtes neurales crâniennes et troncales en début de migration; Pax-3 pour certaines crêtes troncales et une sous-population de crêtes crâniennes, les crêtes cardiaques; Ret et Phox $2 \mathrm{~b}$ pour les crêtes à l'origine du système nerveux entérique; le récepteur du NGF (nerve growth factor) de basse affinité p75 pour les crêtes troncales et entériques; enfin, certains gènes de la famille Hox pour les crêtes neurales rhombencéphaliques (c'est-à-dire provenant du cerveau postérieur). Chez le xénope, ce sont surtout les crêtes neurales crâniennes qui ont fait l'objet d'études et on dispose maintenant comme marqueurs d'ADAM13, une disintégrine, et des facteurs de transcription à doigts de zinc, Zic.

Chez l'oiseau, il existe un autre outil précieux, le facteur de transcription Slug, qui est exprimé par les cellules du primordium des crêtes neurales, et il constitue à ce jour un des marqueurs les plus précoces de cette population [7]. II est exprimé aussi par les crêtes neurales crâniennes en migration non seulement chez le poulet mais aussi chez le xénope. Chez la souris, on dispose de l'équivalent, Snail, un autre facteur de transcription de la même famille. Par ailleurs, le récepteur de type $B$ de I'endothéline-3 s'avère un excellent marqueur des mélanoblastes précoces tant chez la souris que chez le poulet [8]. Grâce à l'apport de la génomique, de nouveaux marqueurs sont progressivement disponibles: cela va d'Id2, un régulateur transcriptionnel sélectivement exprimé par les crêtes neurales crâniennes migratrices et prémigratrices [9], à certaines molécules d'adhérence comme les cadhérines-6B et 7 [10] $\left(\mathrm{m} / \mathrm{s} 2000, n^{\circ} 1\right.$, p. 128), RhoB une petite GTPase associée au cytosquelette [11], en passant par la noéline, un facteur sécrété tout récemment caractérisé [12]. L'intérêt majeur de ces différents marqueurs est qu'ils ont été sélectionnés pour leur expression très spécifique à un moment particulier du développement des crêtes neurales: la spécification, la délamination, la migration, ce qui en fait des outils précieux pour mieux appréhender certaines anomalies acci- dentelles ou induites expérimentalement, par la transgenèse par exemple.

\section{Par où passent-elles? Un code de la route plutôt répressif !}

Les cellules des crêtes neurales ne migrent pas au hasard. Elles quittent le tube neural à un stade bien précis de son développement et se déplacent selon des trajets parfaitement définis tant dans l'espace que dans le temps, qui diffèrent le long de l'axe rostrocaudal. Ainsi les trajets sont-ils sensiblement différents selon que I'on considère les niveaux crânien, cervical, troncal ou sacré. On peut néanmoins dégager des caractéristiques communes à toutes les voies de migration quelles qu'elles soient. Les cellules des crêtes neurales occupent toujours des matrices extracellulaires constituées d'un réseau de fibres de collagènes et de fibronectine et bordées par la lame basale d'épithéliums tels que l'ectoderme, le tube neural ou les somites (figure 2A). Au cours des phases initiales de la migration, les espaces occupés sont restreints, totalement acellulaires et souvent limités par des épithéliums. Une telle organisation spatiale des voies de migration laisse à penser qu'elles constituent des couloirs balisés qui permettent aux cellules de rejoindre leurs territoires cibles: c'est la morphologie des tissus traversés qui dicterait le tracé des voies de migration. Conséquence importante: les multiples directions prises par les cellules sont en grande partie responsables de la ségrégation des lignages issus des crêtes neurales; les cellules émigrant, par exemple, sous l'épiderme donneront les mélanocytes, tandis que celles émigrant le long du tube neural vont fournir le système nerveux périphérique.

Cependant, la structure des voies de migration n'est pas seule cause du choix des routes empruntées par les cellules car certaines régions de I'embryon présentent apparemment nombre des caractéristiques requises pour la migration et, pourtant, ne sont pas colonisées par les cellules des crêtes neurales. Chez les oiseaux et les mammifères, c'est le cas notamment, dans la région troncale, de la notochorde et de la moitié caudale de chaque somite. Les somites sont des unités répétées du mésoderme de 


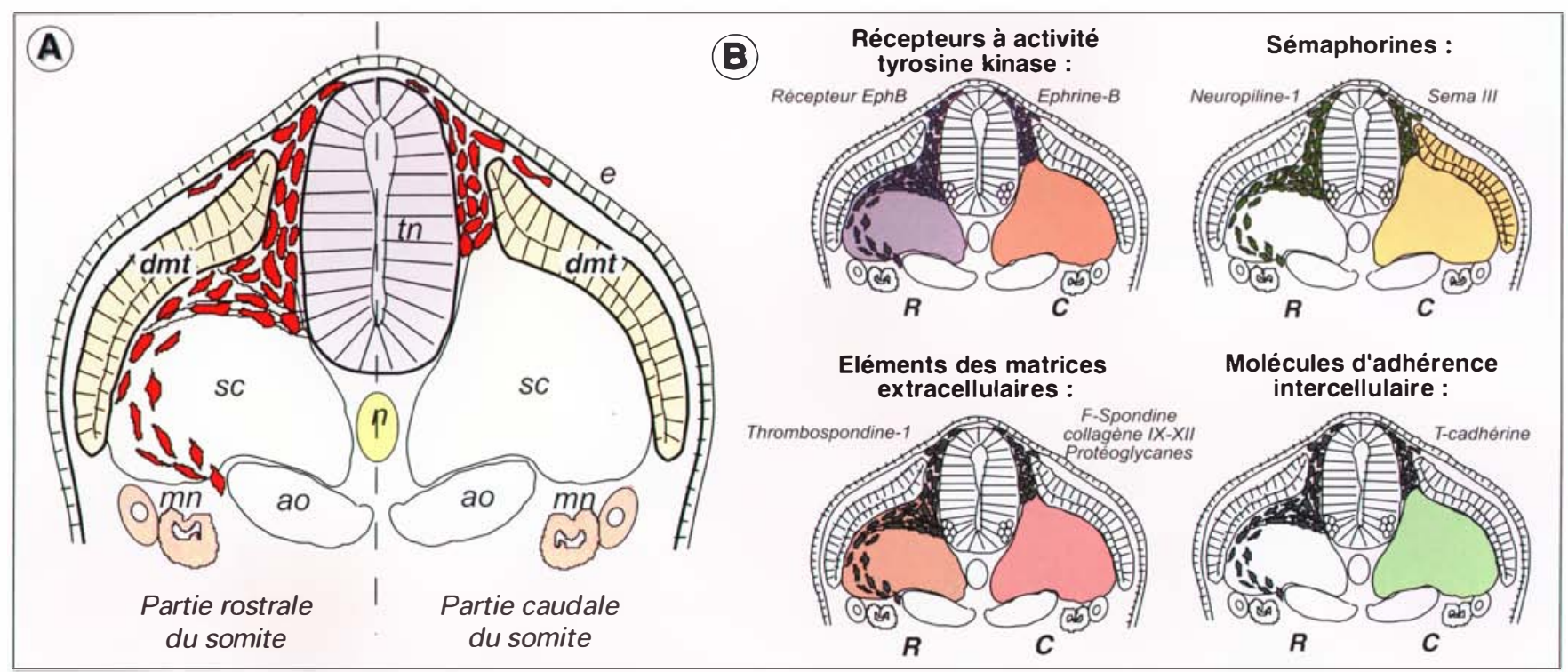

Figure 2. A. Les principales voies de migration des cellules des crêtes neurales dans la région troncale chez l'embryon d'oiseau. Les cellules des crêtes neurales (en rouge), une fois séparées du tube neural (tn), se répartissent tout d'abord entre le tube neural et l'ectoderme (e) puis, plus ventralement, dans une poche entre le tube neural, le dermomyotome (dmt) et le sclérotome (sc) issus du somite. Selon que les cellules font face à la partie rostrale ou caudale du somite, elles vont être bloquées dans leur migration (au niveau caudal, partie droite du schéma) ou au contraire la poursuivre (au niveau rostral, partie gauche du schéma) pour ensuite contourner le sclérotome en longeant la lame basale du myotome pour rejoindre les parties ventrales de l'embryon où elles vont former le ganglion sympathique et la médullosurénale. Certaines s'accumulent le long du tube neural pour former le ganglion sensoriel. Enfin, d'autres cellules vont secondairement envahir l'espace entre l'ectoderme et le dermomyotome pour donner les mélanocytes. II apparaît donc que les cellules des crêtes neurales suivent préférentiellement les lames basales des épithéliums (lignes noires épaisses) et évitent certaines régions comme la notochorde ( $n$ ) et la partie caudale du sclérotome. B. Répartition des molécules guides. Répartition dans les moitiés caudales (C) et rostrales $(R)$ du sclérotome et à la surface des cellules des crêtes neurales de l'éphrine B et de son récepteur EphB, de la sémaphorine III et de son récepteur, la neuropiline, de molécules des matrices extracellulaires (F-spondine, collagènes et protéoglycanes) et de la T-cadhérine. Ao: aorte; mn: mésonéphros.

part et d'autre du tube neural à l'origine du derme, des muscles squelettiques et des vertèbres. Bien que rien ne semble distinguer les moitiés rostrale et caudale du somite sur le plan morphologique, les cellules des crêtes neurales n'envahissent que sa partie rostrale (figure $2 A$ ). Une telle restriction de la migration vise probablement à cloisonner la population de crêtes neurales, qui est au départ répartie uniformément le long du tube neural de façon à constituer des ganglions sensoriels spinaux bien individualisés. Les molécules guides ont été activement recherchées; elles appartiennent à plusieurs catégories: éléments des matrices extracellulaires (F-spondine, collagènes, protéoglycanes), molécules d'adhérence intercellulaire (T-cadhérine), sémaphorines et récepteurs à activité tyrosine kinase de la famille des éphrines [13151. Toutes ces molécules sauf une, la thrombospondine-1, sont réparties dans la moitié caudale du somite et ont pour effet d'empêcher la migration des cellules dans cette région (figure 2B). Ce qui est frappant, c'est la redondance des systèmes inhibiteurs, révélant par là l'importance vitale de la séparation des sous-populations des crêtes neurales en flux distincts.

Néanmoins, on peut se poser la question de l'existence d'un déterminisme précoce des crêtes neurales ou de certaines sous-populations qui les prédestine à se déplacer dans une direction donnée. Le débat est encore ouvert. Certaines expériences de greffe hétérotopique suggèrent que tel n'est pas le cas: les crêtes neurales se comportent plutôt en fonction de l'endroit où elles sont greffées et non pas de leur lieu d'origine. De même, si le tube neural est retourné de $180^{\circ}$ dans le sens dorso-ventral, les cellules "remontent" le chemin [16]. Le propos doit être cependant nuancé: dans la région du cerveau postérieur, les cellules des crêtes neurales en migration expriment le même répertoire de gènes homéotiques de la famille Hox que la région du tube neural d'où elles proviennent, et cela pourrait déterminer à la fois les trajets empruntés et les dérivés obtenus (pour discussion, voir $[17,18])$. De même, il n'est pas exclu que les mélanoblastes issus des crêtes neurales puissent être "programmés" pour se disperser dans la voie sousépidermique [19].

Quels sont les éléments des matrices extracellulaires impliqués dans la migration? Diverses expériences réalisées in vitro ont montré que les cellules des crêtes neurales peuvent se déplacer sur des supports variés de fibronectine, collagènes, laminine et vitronectine. A priori, il semble donc que ces cellules soient assez indifférentes au type de substrat auquel elles sont confrontées, ce qui pourrait leur 
permettre d'envahir de nombreuses régions de l'embryon. Cependant, dans le détail, la situation est plus complexe; la laminine en particulier permet une migration sensiblement plus rapide que les autres composantes matricielles, ce qui pourrait avoir des incidences in vivo. Quoi qu'il en soit, on ne sait toujours pas pour l'heure quels éléments sont effectivement utilisés par les cellules dans l'embryon. On peut penser que toutes ces composantes servent en association pour la migration et que c'est la proportion relative de l'une ou l'autre et leur conformation dans l'espace qui importent. Seule I'analyse détaillée de mutants conditionnels d'embryons dépourvus de l'une ou l'autre des molécules matricielles pourra nous donner une réponse plus précise.

Un certain nombre de points reste encore à élucider. En particulier, il est flagrant que la population des cellules reste groupée en cours de migration. La configuration des voies de migration y contribue très probablement et, notamment, le fait que les cellules migrent très activement sur la laminine pourrait éviter un éparpillement de la population (les lames basales des épithéliums ont une répartition spatiale beaucoup plus restreinte que les matrices de fibronectine-collagène). Ce n'est toutefois pas la seule raison: il est possible que les cellules des crêtes neurales en migration expriment des molécules adhésives spécifiques leur permettant de se reconnaître mutuellement. Ce rôle est-il dévolu par exemple à la cadhérine-7?

\section{Comment migrent-elles? Les intégrines dans le rôle du grand timonier}

Du fait de leur interaction avec les molécules des matrices extracellulaires, d'une part, et de leur capacité à structurer le cytosquelette et à transduire des signaux mécano-chimiques, $d^{\prime}$ autre part, les intégrines constituent le moteur principal de la locomotion cellulaire $\left(m / s\right.$ 1999, $\left.n^{\circ} 5, p .721\right)$. En outre, il est maintenant bien établi qu'elles jouent aussi un rôle dans le contrôle de la prolifération, la différenciation et la mort cellulaire [20]. Les intégrines sont des hétérodimères de chaînes $\alpha$ et $\beta$ [21] et, à I'heure actuelle, on ne compte pas moins de 24 récepteurs différents, dont certains, comme $\alpha 5 \beta 1$ et $\alpha 6 \beta 1$, ne reconnaissent qu'une molécule des matrices, respectivement la fibronectine et la laminine, tandis que d'autres offrent un large éventail de ligands. Inversement, à chaque molécule matricielle correspondent plusieurs intégrines différentes. On est donc confronté à un système très complexe avec une redondance apparente. Est-ce à dire que tous les récepteurs sont équivalents et interchangeables? II est clair maintenant qu'une cellule répondra différemment à une même moléculc des matrices selon qu'elle interagira avec elle par des intégrines distinctes: tantôt, elle se déplacera, tantôt, elle restera immobile; tantôt, elle proliférera, tantôt elle restera quiescente et engagera un processus de différenciation.

La capacité des cellules des crêtes neurales à interagir avec un grand nombre de composantes des matrices extracellulaires se reflète dans la grande variété d'intégrines exprimées au cours de la migration. Ce répertoire varie au cours du temps: chez le poulet, parmi les récepteurs à la laminine, I'intégrine $\alpha 6 \beta 1$ disparaît de la surface des cellules au début de la migration pour ne réapparaître qu'à la différenciation des neurones sensoriels [22]. Inversement, l'intégrine $\alpha 4 \beta 1$, un récepteur à la fibronectine, commence à être exprimée au déclenchement de la migration [23]. Le registre d'intégrines pourrait même avoir une incidence sur le parcours emprunté par la cellule. Si l'on modifie par exemple le patron d'expression d'intégrines dans des cellules de sarcome normalement capables d'utiliser les mêmes voies de migration que les crêtes neurales quand on les injecte dans l'embryon de poulet, celles-ci investissent prématurément la voie sous-épidermique [24]. Les analyses fonctionnelles in vitro montrent que, dans les cellules des crêtes neurales, seules certaines intégrines sont impliquées directement dans les phénomènes $d$ 'adhérence et de motilité. II s'agit, notamment pour la fibronectine, des intégrines $\alpha 4 \beta 1$, $\alpha 8 \beta 1$, et $\alpha v \beta 1[23,25]$. Pour autant, ces intégrines n'ont pas toutes les mêmes fonctions. L'intégrine $\alpha v \beta 1$, par son association avec le cytosquelette d'actine, participerait à l'ancrage transitoire de la cellule pendant le déplacement et aurait un rôle dans la traction. L'intégrine $\alpha 4 \beta 1$, à l'inverse, ne serait pas ancrée au cytosquelette mais serait plutôt impliquée dans la dynamique de locomotion par son activité de signalisation [26]. Ceci suggère que, bien qu'il puisse y avoir une certaine redondance fonctionnelle, comme l'indiquent les expériences d'inactivation de gènes par recombinaison homologue [27], les intégrines possèdent des fonctions définies et coopèrent entre elles durant le déplacement de la cellule. Au cours de la locomotion, l'activité des intégrines ne se borne cependant pas à promouvoir l'adhérence et à régler l'organisation du cytosquelette. En particulier, il est apparu, grâce à des études in vitro, qu'elles répriment l'adhérence intercellulaire relayée par la $\mathrm{N}$-cadhérine au cours de la migration, empêchant ainsi une cohésion trop importante, au sein de la population cellulaire, qui pourrait ralentir le déplacement [28]. Paradoxalement, au moins in vitro, l'intégrine $\alpha 5 \beta 1$ ne semble impliquée ni dans l'adhérence, ni dans la migration, bien qu'elle soit considérée comme le principal récepteur de la fibronectine. Cependant, les souris déficientes en cette intégrine présentent un taux anormalement élevé de figures apoptotiques dans les crêtes neurales en route vers les arcs branchiaux, ce qui suggère qu'elle est essentielle au maintien de la survie des cellules [29]. Les crêtes neurales posséderaient donc un registre étendu d'intégrines en relation avec les différentes missions à accomplir dans un environnement très fluctuant: certaines assureraient un rôle dans l'adhérence et la locomotion, d'autres dans la régulation de I'expression des autres molécules adhésives, d'autres enfin dans la prolifération, la survie et peut-être la différenciation.

\section{Pourquoi migrent-elles? BMP-4 ou l'invitation au voyage}

Au cours des années récentes, des progrès substantiels ont été accomplis dans la compréhension des mécanismes moléculaires qui accompagnent la délamination des crêtes neu- 
rales, principalement au niveau troncal. II ressort que la formation des crêtes neurales n'est qu'un des maillons de la chaîne complexe des événements qui met en place et structure la future moelle épinière selon l'axe dorso-ventral [30]. Parmi les acteurs moléculaires qui interviennent, certains membres de la famille du TGF- $\beta$, les bone morphogenetic proteins 4 et 7 (BMP-4 et BMP-7) [31], produits tout d'abord par l'ectoderme superficiel qui est relayé par le tube neural lui-même, vont tour à tour spécifier l'identité de tous les types cellulaires de la partie dorsale du tube neural, dont les crêtes neurales. Cependant, ces dernières présentent une singularité qui les distingue de toutes les autres cellules du tube neural: ce sont les seules à entreprendre une migration, ce qui suggère qu'elles reçoivent un signal supplémentaire responsable de ce comportement. La recherche d'un tel signal n'a abouti qu'à... BMP-4! En effet, les études réalisées tant in vivo qu'in vitro montrent clairement que BMP-4 seule suffit à provoquer la délamination des crêtes neurales suivie de leur migration $[32,34]$. De plus, dans certaines conditions, elle peut aussi induire la migration des cellules du tube neural qui ne sont pas naturellement destinées à le faire [35]. Plutôt qu'un signal spécifique qui s'ajoute à celui de BMP-4 pour induire la migration des crêtes neurales, il est possible qu'il existe un signal inhibiteur qui restreigne le potentiel migratoire de toutes les cellules du tube neural à l'exception des crêtes neurales, soit directement, soit indirectement, en bloquant I'action de BMP-4. Noggin, un inhibiteur diffusible des BMP, est un candidat de choix car il est en effet exprimé dans le tube neural jusqu'au moment même du début de la migration des crêtes neurales et il est capable de réguler l'action de BMP-4 sur le tube neural [33) (m/s 1994, $\left.n^{\circ} 1, p .84\right)$. Cependant, I'action de Noggin ne peut rendre compte à elle seule du fait que la délamination n'a lieu que dans la partie dorsale du tube neural. D'autres facteurs tels que Sonic hedgehog, qui possède une activité antagoniste des BMP dans la spécification des types cellulaires de la moelle épinière, ou les protéines de la famille Wnt interviennent peut-être dans ce phénomène [6].
Selon les modèles actuels, BMP-4 pourrait agir sur les cellules des crêtes neurales en contrôlant directement ou indirectement l'expression de gènes codant pour des molécules adhésives et des éléments associés au cytosquelette, tous acteurs de la transition épithélio-mésenchymateuse (figure 3). La première molécule d'adhérence intercellulaire exprimée par les cellules du neuro-épithélium est la N-cadhérine; elle persiste sur ces cellules durant l'intégralité du processus d'émigration des crêtes neurales, mais celles-ci la perdent dès lors qu'elles ont entamé leur migration. Cette disparition de la N-cadhérine est supposée rendre irréversible la séparation des crêtes neurales du reste du tube neural. En effet, le maintien de l'expression de la $\mathrm{N}$-cadhérine dans les crêtes neurales après injection d'un vecteur d'expression adénoviral qui code pour cette protéine provoque une accumulation de cellules dans la partie dorsale du tube neural [10]. En revanche, la nature du signal qui réprime l'expression de la $\mathrm{N}$-cadhérine au moment précis de la délamination est inconnue. Après leur spécification, les cellules des primordiums des crêtes neurales dans le tube neural expriment très spécifiquement la cadhérine-6B. Comme la $\mathrm{N}$ cadhérine, elle disparaît de la surface des crêtes neurales dès leur délamination. Sa fonction est inconnue, mais cette expression fugace dans les crêtes neurales, non seulement en fait un marqueur excellent des primor-

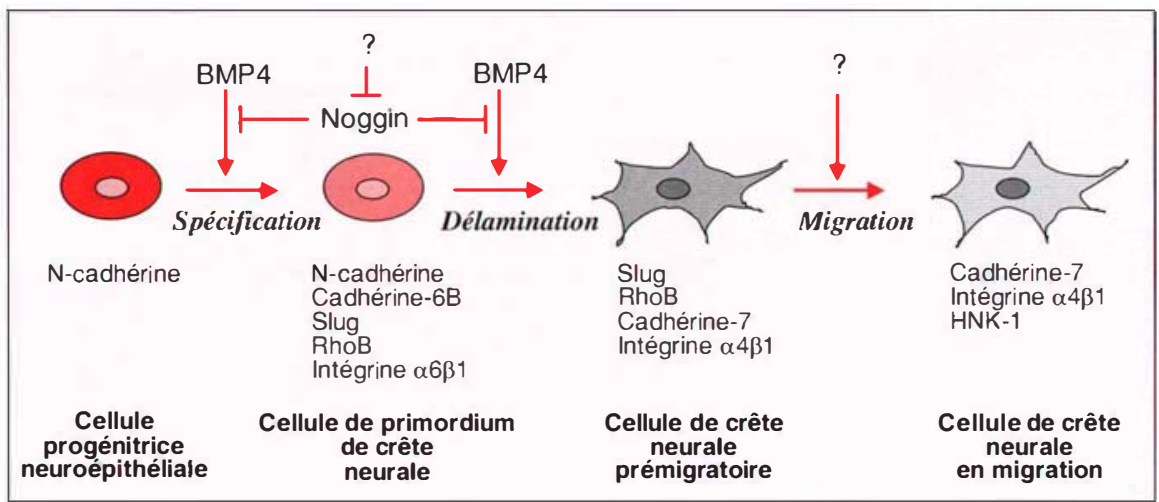

Figure 3. Séquence présumée des événements cellulaires au cours de la spécification, la délamination et la migration des cellules des crêtes neurales. Les étapes contrôlées par BMP-4 sont indiquées, ainsi que les changements importants et rapides dans le répertoire des molécules adhésives (intégrines et cadhérines) et dans l'expression de Slug et de RhoB à chacune des étapes du processus. diums des crêtes neurales, mais suggère aussi qu'elle participe au déterminisme de cette population. A la place des cadhérines $\mathrm{N}$ et $6 \mathrm{~B}$, les crêtes neurales en migration expriment la cadhérine-7 probablement aussi sous l'effet de BMP-4. II est à noter que l'expression de la cadhérine-7 ne semble pas a priori incompatible avec la locomotion car elle présente une affinité de liaison beaucoup plus faible que la $\mathrm{N}$-cadhérine 136]. A côté des cadhérines, RhoB constitue indubitablement une des cibles les plus intéressantes de BMP4. Il s'agit d'une GTPase responsable de l'organisation du cytosquelette $d^{\prime}$ actine dans la cellule, qui participe directement à la délamination des crêtes neurales mais pas, semble-t-il, à leur migration [11]. On sait encore peu de choses, en revanche, sur le contrôle de l'expression et de l'activité des intégrines au cours de la délamination des crêtes neurales, si ce n'est que l'intégrine $\alpha 6 \beta 1$ disparaît au profit $\mathrm{d}^{\prime} \alpha 4 \beta 1$ nécessaire au déplacement. Néanmoins, il est fort possible que ce soit aussi BMP-4 qui contrôle ce changement du répertoire d'intégrines, étant donné que certains membres de la famille du TGF- $\beta$ peuvent expérimentalement accroître l'adhérence des cellules des crêtes neurales à la matrice extracellulaire [32].

Le facteur de transcription Slug est-il un intermédiaire entre BMP-4 et les molécules adhésives, responsable du départ en migration des crêtes neu- 
rales? L'inhibition de l'expression de Slug par des oligonucléotides antisens abolit l'émergence des crêtes neurales, ce qui suggère que ce pourrait être le cas [7]. Cependant, diverses expériences récentes indiquent que Slug n'est pas sous le contrôle direct de BMP-4 [33] mais plutôt de la noéline-1 [12]. Quelles sont les cibles moléculaires de Slug? Pour I'heure, seule une activité de répression de l'expression génique a pu être attribuée aux membres de la famille Snail. En particulier, il a été montré tout récemment chez la souris que Snail réprime l'expression du gène de la $E$-cadhérine $[37,38]$. Or celle-ci est rencontrée dans l'ectoderme mais pas dans le neuroépithélium qui, à l'inverse, exprime la $\mathrm{N}$ cadhérine. La frontière entre le domaine d'expression de la E-cadhérine et celui de la $\mathrm{N}$-cadhérine constitue probablement la zone de détermination des cellules des crêtes neurales. II est donc possible que Slug, en réprimant la E-cadhérine, définisse la population du primordium des crêtes neurales, mais cela reste encore à prouver. En effet, il n'est pas établi que la spécification et la formation des crêtes neurales requièrent nécessairement la perte de l'expression de la E-cadhérine.

\section{Conclusions}

Si les modèles de migration tirés des cellules embryonnaires, comme celui des crêtes neurales, nous apportent peu sur les moteurs moléculaires à I'échelle de la cellule, ce sont en revanche de précieux systèmes pour la compréhension des mécanismes de régulation qui interviennent dans le déclenchement de la migration et le contrôle de la directionalité des cellules. Cela est d'autant plus vrai que le déroulement des étapes de migration des crêtes neurales n'est pas sans rappeler le processus de dissémination de cellules cancéreuses à partir d'une tumeur: perte d'un état cohésif, migration au travers d'une matrice extracellulaire et au sein de tissus conjonctifs, et regroupement dans des sites éloignés du tissu d'origine. II y a donc, tant dans les cellules cancéreuses que dans les cellules des crêtes neurales, nécessité de changement dans I'adhérence cellulaire et adaptation et survie dans des environne- ments tissulaires très divers. Dans les cellules cancéreuses, cependant, on ne peut analyser les étapes précoces de la dissémination des cellules à partir de la tumeur parce que c'est un phénomène par nature aléatoire et rare. Le caractère parfaitement prévisible et reproductible de la migration des crêtes neurales permet donc $d$ 'analyser cette étape et de rechercher la fonction de gènes exprimés au moment du départ en migration des cellules ou d'en caractériser de nouveaux. A cet égard, la découverte du gène Slug est parfaitement démonstrative de l'intérêt que présentent des modèles de migration comme les crêtes neurales. En effet, il a été montré récemment que Snail est impliqué dans la répression de la transcription du gène de la $E$-cadhérine, un gène suppresseur de tumeur, au cours de la progression tumorale chez la souris et chez l'homme. De ce fait, il peut être considéré comme un proto-oncogène $[37,38]$. Ce sont les observations réalisées initialement sur l'embryon qui ont conduit à cette découverte

\section{Remerciements}

Merci à Muriel Altabef et Sandrine Testaz pour leurs commentaires sur le manuscrit. Merci aussi à tous ceux de l'équipe, Muriel Altabef, Nathalie Desban, Artem Jarov, Sandrine Testaz et Claire Thibault, pour leur entrain, leur enthousiasme et leur gaieté. Les projets de l'équipe ont reçu le soutien du Cnrs, de l'Université Pierre-et-Marie-Curie, I'ARC, la Fondation pour la recherche médicale, et la Ligue contre le cancer.

\section{RÉFÉRENCES}

1. Le Douarin NM, Kalcheim C. The neural crest. $2^{\text {nd }}$ ed. New York: Cambridge University Press, 1999.

2. Le Douarin NM. A biological cell labelling technique and its use in experimental embryology. Dev Biol 1973; 30: 217-22.

3. Bronner-Fraser M, Fraser S. Developmental potential of avian trunk neural crest cells in situ. Neuron 1989;3:755-66.

4. Serbedzija GN, Bronner-Fraser M, Fraser SE. Developmental potential of trunk neural crest cells in the mouse. Development 1994; 120:1709-18.

5. Tucker GC, Delarue M, Zada S, Boucaut JC, Thiery JP. Expression of HNK-1/NC-1 epitope in early vertebrate neurogenesis. Cell Tissue Res 1988; 251: 457-65.
6. Ikeya $M$, Lee SMK, Johnson JE, McMahon AP, Takada S. Wnt signalling required for expansion of neural crest and CNS progenitors. Nature 1997; 389: 966-70.

7. Nieto MA, Sargent MG, Wilkinson DG, Cooke J. Control of cell behavior during vertebrate development by Slug, a zinc finger gene. Science $1994 ; 264: 835-9$.

8. Shin MK, Levorse JM, Ingram RS, Tilghman $S M$. The temporal requirement for endothelin receptor-B signalling during neural crest development. Nature 1999; 402 : 496-501.

9. Martinsen BJ, Bronner-Fraser M. Neural crest specification regulated by the helixloop-helix repressor Id2. Science 1998; 281: $988-91$.

10. Nakagawa S, Takeichi M. Neural crest emigration from the neural tube depends on regulated cadherin expression. Development 1998; 125:2963-71.

11. Liu JP, Jessel TM. A role for rhoB in the delamination of neural crest cells from the dorsal neural tube. Development 1998; 125: 5055-67.

12. Barembaum $M$, Moreno $T$, LaBonne $C$, Sechrist J, Bronner-Fraser $M$. Noelin-1 is a secreted glycoprotein involved in generation of the neural crest. Nat Cell Bio $2000 ; 2$ : 219-25.

13. Debby-Brafman A, Burstyn-Cohen $T$, Klar A, Kalcheim C. F-spondin, expressed in somite regions avoided by neural crest cells, mediates inhibition of distinct somite domains to neural crest migration. Neuron $1999 ; 22: 475-88$.

14. Krull CE, Lansford R, Gale NW, et al. Interactions of $\mathrm{EpH}$-related receptors and ligands confer rostrocaudal pattern of trunk neural crest migration. Curr Biol 1997; 7: $571-80$.

15. Eickholt BJ, Mackenzie SL, Graham A Walsh FS, Doherty P. Evidence for collapsin-1 functioning in the control of neural crest migration in both trunk and hindbrain regions. Development 1999; 126: 2181-9.

16. Bronner-Fraser M. Neural crest cell migration in the developing embryo. Trends Cell Biol 1993; 3: 392-7.

17. Couly $G$, Grapin-Botton A, Coltey $P$, Ruhin B, Le Douarin NM. Determination of the identity of derivatives of the cephalic neural crest: incompatibility between How gene expression and lower jaw develop ment. Development 1998; 128: 3445-59.

18. Trainor P, Krumlauf R. Plasticity in mouse neural crest cells reveals a new patterning role for cranial mesoderm. Nat Cell Biol 2000; 2 : 96-102.

19. Erickson CA, Goins TL Avian neural crest cells can migrate in the dorsolateral path only if they are specified as melanocytes. Development 1995; 121: 915-24.

20. Giancotti FG. Integrin signaling: specificity and control of cell survival and cell cycle progression. Curr Opin Cell Biol 1997; 9:691-700. 


\section{RÉFÉRENCES}

21. Hynes RO, Lander AD. Contact and adhesive specificities in the associations, migrations, and targeting of cells and axons. Cell 1992; 68: 303-22.

22. Bronner-Fraser $M$, Artinger $M$, Muschler J, Horwitz AF. Developmentally regulated expression of $\alpha 6$ integrin in avian embryos. Development 1992; 115: 197-211.

23. Kil SH, Krull CE, Cann G, Clegg D Bronner-Fraser $M$. The $\alpha 4$ subunit of integrin is important for neural crest cell migration. Dev Biol 1998; 202 : 29-42.

24. Beauvais A, Erickson CA, Goins T, et al Changes in the fibronectin-specific integrin expression pattern modify the migratory behavior of sarcoma S180 cells in vitro and in the embryonic environment. / Cell Biol 1995; 128: 699-713.

25. Testaz S, Delannet M Duband JL. Adhesion and migration of avian neural crest cells on fibronectin require the cooperating activites of multiple integrins of the $\beta 1$ and $\beta 3$ families. / Cell SCi 1999; 112:4715-28.

26. Liu S, Thomas SM, Woodside DG, et al Binding of paxillin to $\alpha 4$ integrins modifies integrin-dependent biological responses Nature 1999; 402: 676-81.

27. Hynes RO. Targeted mutations in cell adhesion genes: what we have learned from them? Dev Biol 1996; 180: 402-12.

28. Monier-Gavelle F, Duband JL. Cross-talk between adhesion molecules: control of $\mathrm{N}$ cadherin activity by intracellular signals elicited by $\beta 1$ and $\beta 3$ integrins in migrating neural crest cells. / Cell Biol 1997: $137^{\circ}$ 1663-81.

29. Goh KL, Yang JT, Hynes RO. Mesodermal defects and cranial neural crest apoptosis in $\alpha 5$ integrin-null embryos. Development $1997 ; 124: 4309-19$.
30. Lee KJ, Jessell TM. The specification of dorsal cell fates in the vertebrate nervous system. Annu Rev Neurosci 1999; 22: 261 94.

31. Sautier IM Forest $N$. Les protéines de la morphogenèse osseuse BMP. Med Sci 1996 ; $12: 364-70$

32. Delannet $M$, Duband JL. Transforming growth factor- $\beta$ control of cell-substratum adhesion during avian neural crest cell emigration in vitro. Development 1992; 116: 275-87.

33. Sela-Donenfeld D, Kalcheim C. Regulation of the onset of neural crest migration by coordinated activity of BMP4 and Noggin in the dorsal neural tube. Development 999 ; 126: 4749-62.

34. Liem KF, Tremml G, Roelink H, Jessell $T M$. Dorsal differentiation of neural plate cells induced by BMP-mediated signals from epidermal ectoderm. Cell 1995; 82: 969. 79 .

35. Liem KF, Tremml G, Jessell TM. A role for the roof plate and its resident TGF $\beta$-related proteins in neuronal patterning in the dorsal spinal cord. Cell 1997; 91 : 127-38.

36. Dufour S, Beauvais-Jouneau A, Delouvée $A$, Thiery JP. Differential function of $N$ cadherin and cadherin-7 in the control of embryonic cell motility. J Cell Biol 1999; 146:501-16.

37. Batlle E, Sancho E, Franci C, et al. The transcription factor Snail is a repressor of Ecadherin gene expression in epithelial tumor cells. Nat Cell Biol $2000 ; 2$ : 84-9.

38. Cano A, Pérez-Moreno MA, Rodrigo 1, et al. The transcriptional factor Snail controls epithelial-mesenchymal transitions by repressing E-cadherin expression. Nat Cell Biol $2000 ; 2: 76-83$

\section{ms $200(0)$}

\section{Summary}

The odyssey of the neural crest

During embryonic development, massive displacements of cell populations participate in the shaping of the body plan and facilitate the segregation of distinct lineages arising from a population of precursors or, conversely, the regroupment of cells originating from different areas of the body, thereby allowing concerted differentiation programs to occur. For example, in all vertebrates, neural crest cells undergo an extensive migration from the developing brain and spinal cord up to various regions of the body such as the gut, the limbs, and the face where they give rise to a wide array of cell types and tissues. Studies on neural crest cell migration have necessited the development of strategies, such as chick-quail chimeras and dye labeling, and the search for markers, including the HNK-1 epitope, to trace cells on their way to their final destination. In addition, they have provided interesting informations on the mechanism of cell locomotion driven by integrins, a family of receptors of the extracellular matrix, and allowed the discovery of genes essential for the triggering and spatio-temporal control of cell migration. Most importantly, one of these genes, Snail, has been found recently to play a key role in the acquisition of metastatic potential at late stages of epithelial tumor progression, thereby emphasizing the parallel existing between migration of normal embryonic cells and dissemination of cancer cells.

Web : http://www.inra.fr/clermont/mag2000

Pour information, contacter: Yves Rayssogioer

INRA, Clermont-Ferrand - Theix, F-63122 Saint-Genès-Champanelle, France Fax : 33 (0)4 73624638 -E-mail : mag2000@clermont.inra.fr 